\title{
Analysis of a Queuing Model for Slotted Ring Networks
}

\author{
Bart VAN AREM and Erik A. VAN DOORN \\ University of Twente, Faculty of Applied Mathematics, P.O. Box \\ 217, 7500 AE Enschede, Netherlands
}

\begin{abstract}
We study a multi-server multi-queue system which is intended to model a local area network with slotted ring protocol. Two special cases of the model are analysed and the results are used to motivate an approach to approximate mean queue lengths in the general model.
\end{abstract}

Keywords. Multi-server multi-queue system, slotted ring network, local area network, queue length, approximations.

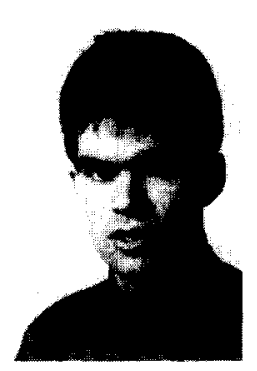

Bart van Arem received the M.S. and Ph.D. degrees in Applied Mathematics in 1986 and 1990 respectively, from the University of Twente, Enschede, The Netherlands. From 1986 until 1990 he was with the University of Twente, doing his $\mathrm{Ph} . \mathrm{D}$. research on queuing models for slotted transmission systems. His research interests include queuing theory, performance evaluation of computer and communication systems and traffic theory.

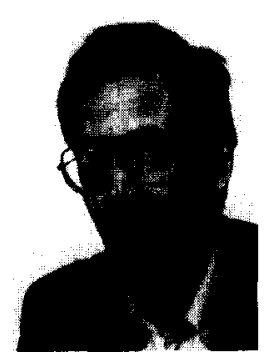

Erik A. van Doorn received his M.S. degree in Mathematics from Eindhoven University of Technology in 1974 and his Ph.D. degree in Technical Sciences from the University of Twente, Enschede, in 1979. From 1980 to 1982 he was with the Neher Laboratories of the Netherlands Postal and Telecommunications Services in Leidschendam, and from 1982 to 1985 with the Centre for Mathematics and Computer Science in Amsterdam. In 1985 he joined the Faculty of Mathematics of the University of Twente again as an associate professor in applied probability. His research interests range from theoretical issues in analysis and probability theory to application-oriented topics in the areas of queuing and teletraffic theory.

\section{North-Holland}

Computer Networks and ISDN Systems 20 (1990) 309-314

\section{Introduction}

The following queuing system generalizes earlier proposals for modelling a local area network with slotted ring protocol. Consider $c$ equidistant slots (servers) moving with constant speed along a closed track. The time needed by a slot to make a complete round trip along the track is denoted by $\tau$. At fixed positions along the track are $n$ stations (queues) numbered $1,2, \ldots, n$, at which packets (customers) arrive in batches. The arrival of batches at station $i$ is governed by a Poisson process with intensity $\lambda_{i}$. The sizes of the batches arriving at station $i$ constitute a sequence of mutually independent and identically distributed random variables with finite means and variances; the batch sizes are also independent of the batch arrival processes. We let $A_{i}$ denote the generic number of packets that arrive at station $i$ in an interval of time of length $\tau / c$ (the time between the passages of two consecutive slots at a particular station). Defining $\mathscr{B}_{i}(z) \equiv E\left(z^{B_{i}}\right), \quad|z| \leqslant 1$, where $B_{i}$ denotes the size of a generic batch arriving at station $i$, and $\mathscr{A}_{i}(z) \equiv E\left(z^{A_{i}}\right),|z| \leqslant 1$, it is easy to see that

$\mathscr{A}_{i}(z)=\exp \left(-\left(\lambda_{i} \tau / c\right)\left(1-\mathscr{B}_{i}(z)\right)\right)$.

By $\alpha_{i}$ and $\sigma_{i}^{2}$ we denote the mean and variance, respectively, of the number of packets that arrive at station $i$ in an interval of time of length $\tau / c$. Clearly,

$\alpha_{i}=\mathscr{A}_{i}^{\prime}(1)=\left(\lambda_{i} \tau / c\right) \mathscr{B}_{i}^{\prime}(1)$

and

$$
\begin{aligned}
\sigma_{i}^{2} & =\mathscr{A}_{i}^{\prime \prime}(1)+\alpha_{i}-\alpha_{i}^{2} \\
& =\left(\lambda_{i} \tau / c\right)\left(\mathscr{B}_{i}^{\prime \prime}(1)+\mathscr{B}_{i}^{\prime}(1)\right),
\end{aligned}
$$

and both $\alpha_{i}$ and $\sigma_{i}^{2}$ are finite by our assumption that the mean and variance of a batch size are finite.

Each station has infinite capacity for storing packets. The packets are released by the stations according to the following mechanism. Any station that has at least one packet in store waits 
until an empty slot comes along and then places a packet in the slot. The full slot subsequently makes a complete round trip along the track, after which it disposes of the packet at the source station and proceeds empty to the next downstream station. Upon returning to its source station a packet disappears from the system.

The models proposed in [12] and [13] are less general than the model described above since batch sizes are assumed to be geometrically distributed in [13] (see also [14]), while packets are supposed to arrive singly in [12]. The model studied in [9] has the additional feature that each station can have at most one packet on the ring at any time.

Throughout this paper we will assume that steady-state conditions prevail, implying in particular that the stability condition

$\sum_{i=1}^{n} \alpha_{i}+\max _{i \leqslant j \leqslant n}\left(\alpha_{j}\right)<1$

(see [13] and, for a formal proof, [1]) is satisfied. We let $L_{i}$ denote the mean (steady-state) queue length at an arbitrary point in time and $W_{i}$ the mean (steady-state) packet delay at station $i, i=$ $1,2, \ldots, n$. In what follows our interest focuses on the mean queue lengths, but since, by Little's formula,

$W_{i}=(\tau / c) L_{i} / \alpha_{i}$,

an exact or approximate expression for $L_{i}$ immediately yields a similar result for $W_{i}$.

In this paper we shall first discuss two special cases of the model, viz., the single-slot model in Section 2 and the single-station model in Section 3. Then, in Section 4, we shall indicate how the results for these special cases and the insights gained from these results suggest an approach to approximate $L_{i}$, in the general case. A detailed elaboration of the approach and extensive numerical comparisons will be reported in a later paper.

\section{The Single-slot Model}

If $c=1$, that is, there is only one slot, then the queuing system described in Section 1 can be interpreted as a cyclic-server system with nonzero switch-over times and 1-limited service, the latter meaning that at most one customer is served during a visit of the server to a station. Indeed, after the slot has been occupied by a packet from a particular station, it makes a complete round trip along the track in the full state; but since the slot is not available to other stations during this round trip, we may as well assume that the slot remains at the station to perform a service of length $\tau$ before it proceeds empty to the next downstream station.

Only in some exceptional cases explicit analytical results are available for cyclic-server systems, even if the quantities of interest are mean queue lengths as in our case. However, useful information can be obtained from the recently discovered so-called pseudo-conservation laws, which are exact expressions for weighted sums of the mean waiting times (or, cf. (1.5), of the mean queue lengths). The very general pseudo-conservation law of Boxma and Groenendijk [3, formula (5.8)] allows batch Poisson arrival processes and therefore serves our purposes. Translating their result into our context gives us the following theorem.

Theorem 2.1. If $c=1$, the mean queue lengths $L_{i}$, $i=1,2, \ldots, n$, satisfy the relation

$$
\begin{aligned}
& \sum_{i}\left(1-\alpha_{i}-\sum_{j} \alpha_{j}\right) L_{i} \\
& =\sum_{i} \sigma_{i}^{2}+\frac{1}{2}\left(\sum_{i} \alpha_{i}^{2}+\left(\sum_{i} \alpha_{i}\right)^{2}-\sum_{i} \alpha_{i}\right) .
\end{aligned}
$$

Note that the switch-over times between consecutive stations, which are determined by the relative positions of the stations along the track, do not occur in (2.1).

The next two corollaries contain results for situations in which the exact mean queue length(s) can be obtained directly from Theorem 2.1.

Corollary 2.2. If $c=1$ and $n=1$, then, dropping the index $i=1$,

$L=\frac{\boldsymbol{\sigma}^{2}+\alpha^{2}-\frac{1}{2} \alpha}{1-2 \alpha}$.

Corollary 2.3. If $c=1$ and the system is completely symmetrical (implying in particular that $\alpha_{i} \equiv \alpha$ and $\left.\sigma_{i}^{2} \equiv \sigma^{2}, i=1,2, \ldots, n\right)$, then

$L_{i}=\frac{\sigma^{2}+\frac{1}{2}(n+1) \alpha^{2}-\frac{1}{2} \alpha}{1-(n+1) \alpha}, \quad i=1,2, \ldots, n$.

In [13, formula (17)] Zafirovic-Vukotic and Niemegeers, referring to earlier and more general 
results in the literature, give an expression for $W_{i}$ in the symmetric context of Corollary 2.3. Apparently, their formula contains an error $\left(\gamma^{3}\right.$ should be $\gamma^{-1}$ ).

In the cases that are not covered by Corollaries 2.2 and 2.3 no exact expressions for $L_{i}$ are available. However, several suggestions for approximations for $L_{i}$ have appeared in the literature, see $[4,6-8,10,11]$; of these only [10] allows batch arrivals. Since, in the case of single arrivals, the approximation of $[10]$ is generally inferior to those of $[4,6-8,11]$, it seems worthwhile to try to adapt the approximate assumptions in the latter references to batch arrivals. Choosing the approach in [4] this means that for each station $i$ we must take into account an additional term representing the expected number of packets an arbitrary packet arriving at station $i$ finds in front of it in its batch. It is not difficult to show, see [10], that this quantity equals

$\frac{1}{2} \mathscr{B}_{i}^{\prime \prime}(1) / \mathscr{B}_{i}^{\prime}(1)=\frac{1}{2}\left(\left(\sigma_{i}^{2} / \alpha_{i}\right)-1\right)$.

With this information the generalization to batch arrivals of the arguments in [4] is straightforward. It results in the approximation

$$
\begin{aligned}
L_{i} \approx & \frac{\sigma_{i}^{2}-\alpha_{i}}{1-\alpha_{i}-\sum \alpha_{j}} \\
& +\frac{\alpha_{i}}{2} \frac{1+\alpha_{i}-\sum \alpha_{j}}{1-\alpha_{i}-\sum \alpha_{j}} \frac{\sum \alpha_{j}+\left(\sum \alpha_{j}\right)^{2}+\sum \alpha_{j}^{2}}{\sum \alpha_{j}-\left(\sum \alpha_{j}\right)^{2}+\sum \alpha_{j}^{2}} .
\end{aligned}
$$

The first term in the right-hand side of (2.4) is due to batches; indeed, if packets arrive singly at station $i$, then $\sigma_{i}^{2}=\alpha_{i}$.

The approximation (2.4) may be slightly improved by copying the minor refinement of the approximation in [4] suggested in [6]. The adaptation to batch arrivals of the approximation procedures suggested in [7], [8] and [11] seems possible, but we have not worked out the details.

\section{The Single-station Model}

Since we consider the model with only one station $(n=1)$ in this section, we suppress the index $i=1$.

In [2] an algorithmic procedure is presented for calculating the generating function of the distribu-

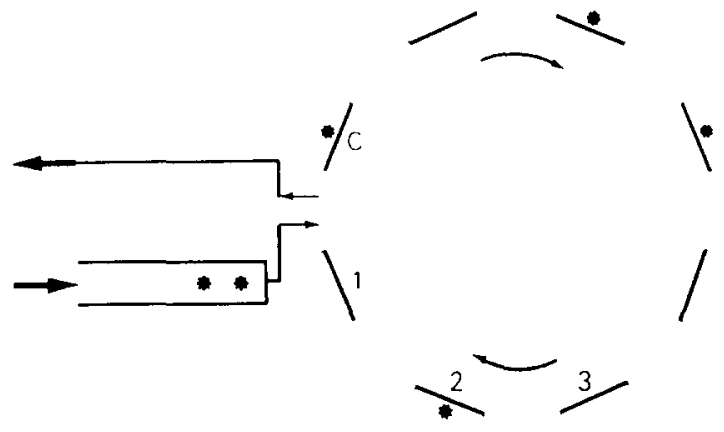

Fig. 1.

tion of the (steady-state) queue length at the station, embedded at instants just before a slot passes the station (passage instants). Specifically, the state of the system at a passage instant can be described by a vector $(Q, D)$, where $Q$ denotes the queue length at the station, $D=\left(D^{(1)}, D^{(2)}, \ldots, D^{(c)}\right)$ and $D^{(j)}$ is 0 or 1 according to whether the $j$ th slot is empty or occupied, respectively, at a passage instant. Here slots are numbered counterclockwise (assuming that the slots move clockwise) starting with the slot which is about to visit the station (see Fig. 1). Evidently, the successive states of the system at passage instants constitute a Markov chain. Moreover, it is shown in [2] that this Markov chain is irreducible and aperiodic if and only if $\operatorname{GCD}\{b, c\}=1$, where $b \equiv \operatorname{GCD}\{j \in$ $\mathbb{N} \mid \operatorname{Pr}\{B=j\}>0\}$. We assume that this condition is satisfied. Now writing $Q(z, \delta) \equiv E\left(z^{Q} I(D=\right.$ $\delta)),|z| \leqslant 1, \delta \in \Delta$, where $\Delta=\{0,1\}^{\mathrm{c}}$ and $I(F)$ denotes the indicator function of the event $F$, it is shown in [2] that the functions $Q(z, \delta), \delta \equiv$ $\left(\delta_{1}, \delta_{2}, \ldots, \delta_{c}\right) \in \Delta$, satisfy

$$
\begin{aligned}
z^{\delta} Q(z, \delta)=\mathscr{A}(z) & (Q(z, f(\delta)) \\
+ & \left.\left(1-2 \delta_{c}\right) Q(0, h(\delta))\right),
\end{aligned}
$$

where

$f(\delta) \equiv\left(1-\delta_{c}, \delta_{1}, \delta_{2}, \ldots, \delta_{c-1}\right)$,

$h(\delta) \equiv\left(0, \delta_{1}, \delta_{2}, \ldots, \delta_{c-1}\right)$.

The main effort in [2] consists in solving the equations (3.1) for the generating functions $Q(z, \delta)$ and determining the unknown constants $Q(0, h(\delta))$. Once $Q(z, \delta)$ is known for each $\delta \in \Delta$, the generating function $Q(z)$ of the distribution of the queue length embedded at passage instants can be obtained by summing over all $\delta \in \Delta$.

In principle one can obtain the expected queue length $E Q$ by differentiating $Q(z)$ and letting 
$z \uparrow 1$. A short cut to this procedure is described and illustrated with numerical results in [2]. The algorithm is numerically feasible for $c \leqslant 7$.

We will now derive a representation for $E Q$ which does not lead directly to explicit results but yields additional insight. By applying Little's formula to the part of the track which precedes the station and has length $l / c$ (where $l$ is the length of the track), we find that a slot which is about to pass the station has probability $\alpha$ of being full. Since the state of a slot is constant between passage times it follows that

$$
\begin{aligned}
\operatorname{Pr}\left\{D^{(j)}=1\right\} & =1-\operatorname{Pr}\left\{D^{(j)}=0\right\} \\
& =\alpha, \quad j=1,2, \ldots, c .
\end{aligned}
$$

Taking $z=1$ and $\delta_{c}=0$ in (3.1) and summing the resulting equations over all values of $\left(\delta_{1}, \delta_{2}, \ldots\right.$, $\left.\delta_{c-1}\right)$ we get

$\operatorname{Pr}\left\{D^{(c)}=0\right\}=\operatorname{Pr}\left\{D^{(1)}=1\right\}+\frac{1}{2} \sum_{\delta \in \Delta} Q(0, h(\delta))$.

Now combining (3.2) and (3.3) we conclude

$$
\begin{aligned}
\frac{1}{2} \sum_{\delta \in \Delta} Q(0, h(\delta)) & =\operatorname{Pr}\left\{Q=0, D^{(1)}=0\right\} \\
& =1-2 \alpha .
\end{aligned}
$$

Next, differentiating (3.1) and letting $z \uparrow 1$ yields

$$
\begin{aligned}
\delta_{c} Q(1, \delta)+Q^{\prime}(1, \delta) \\
=Q^{\prime}(1, f(\delta)) \\
\quad+\mathscr{A}^{\prime}(1)(Q(1, f(\delta)) \\
\left.\quad+\left(1-2 \delta_{c}\right) Q(0, h(\delta))\right) .
\end{aligned}
$$

Summing the preceding equations over all $\delta \in \Delta$ such that $\delta_{c}=1$ and substituting (3.2) and (3.4) gives

$$
\begin{aligned}
(1-2 \alpha) E Q= & \operatorname{cov}\left(Q, D^{(c)}\right)+\operatorname{cov}\left(Q, D^{(1)}\right) \\
& +\alpha(1-\alpha) .
\end{aligned}
$$

Subsequently differentiating (3.1) twice, letting $z \uparrow 1$ and summing over all $\delta \in \Delta$, we get

$\operatorname{cov}\left(Q, D^{(c)}\right)=\frac{1}{2}\left(\sigma^{2}-\alpha+\alpha^{2}\right)$.

Combining (3.5) and (3.6) finally gives

$E Q=\frac{\sigma^{2}+\alpha-\alpha^{2}+2 \operatorname{cov}\left(Q, D^{(1)}\right)}{2(1-2 \alpha)}$,

which is the representation we were aiming at. Since packets arrive according to a batch Poisson process while the arrival (and hence departure) rate of packets is $\alpha c / \tau$, the mean queue length at an arbitrary point in time $L$ satisfies $L=E Q$ $-\frac{1}{2}(\alpha c / \tau)(\tau / c)=E Q-\frac{1}{2} \alpha$. Hence (3.7) leads to

$L=\frac{\sigma^{2}+\alpha^{2}+2 \operatorname{cov}\left(Q, D^{(1)}\right)}{2(1-2 \alpha)}$.

We note that the result of Corollary 2.2 can be regained by combining (3.6) and (3.8).

In what follows we study the behaviour of $L \equiv L(c)$ as a function of $c$, while keeping $\tau / c$, and hence $\alpha$ and $\sigma^{2}$, constant. First note that $D^{(1)}$ in (3.8) represents the state of a slot which is about to pass the station. But the most recent point in time at which the state of this particular slot could have been changed occurred $c$ passage instants earlier. It is therefore very likely that $\operatorname{cov}\left(Q, D^{(1)}\right)$ goes to zero as $c$ goes to infinity. So, in view of (3.8), we state the following.

Conjecture 3.1. $L(c) \rightarrow \frac{1}{2}\left(\sigma^{2}+\alpha^{2}\right) /(1-2 \alpha)(c \rightarrow$ $\infty)$.

Surprisingly, the conjectured limit equals the mean queue length one would obtain if the states of the successive slot passing the station would be independent (which is not true) and equal to 1 with probability $\alpha$ (which is true), as is shown in the Appendix.

There is also evidence supporting the following.

Conjecture 3.2. $L(c+1)<L(c), c=1,2, \ldots$

First, with the explicit results obtained in [2] it is not difficult to prove that $L(2)<L(1)$. Secondly, for the same reasons that led to Conjecture 3.1 it is in fact plausible that $\operatorname{cov}\left(Q, D^{(1)}\right)$ goes to zero monotonically, which, in view of (3.8) and the fact that $L(2)<L(1)$, is equivalent to the statement in Conjecture 3.2. Thirdly, the exact results for $c \leqslant 5$ as well as the simulation results for $c=30$ reported in [2] support Conjecture 3.2. Finally, it is known for certain many-server models like $\mathrm{G} / \mathrm{M} / c$ and $\mathrm{E}_{n} / \mathrm{D} / c$ that increasing the number of servers while keeping the load per server constant makes that the mean queue length decreases, cf. [5]. These results suggest that the same may be true for other models, in particular the one at hand. 
Summarizing the preceding we believe that there is strong evidence indicating that $L(c)$ is a decreasing function of $c$ whose limit as $c$ goes to infinity equals the mean queue length one would have if the states of the successive slots passing the station would be independent and equal to 1 with probability $\alpha$. For completeness' sake we note that $L(1)$ is indeed larger than the conjectured limit of $L(c)$ as $c \rightarrow \infty$, for, by $(2.2)$,

$$
\begin{aligned}
L(1)-\frac{\sigma^{2}+\alpha}{2(1-2 \alpha)} & =\frac{\sigma^{2}+\alpha^{2}-\alpha}{2(1-2 \alpha)} \\
& =\frac{E\left(A^{2}\right)-E(A)}{2(1-2 \alpha)}>0 .
\end{aligned}
$$

\section{The General Model}

In the light of the difficulties we have encountered in the exact analysis of the single-slot and single-station models it seems illusory to try to obtain exact expressions for $L_{i}, i=1,2, \ldots, n$, in the general model of Section 1 . Consequently approximations are called for.

As a basis for approximations we would suggest to extrapolate to the multi-station case the conjectured behaviour of $L(c)$ in the single-station case. That is, viewing $L_{i} \equiv L_{i}(c), \quad i=$ $1,2, \ldots, n$, as a function of $c$, while keeping $\tau / c$, and hence $\alpha_{j}$ and $\sigma_{j}^{2}, j=1,2, \ldots, n$, constant, we conjecture that $L_{i}(c)$ is a decreasing function of $c$ whose limit as $c \rightarrow \infty$ equals the mean queue length one would have at station $i$ if the states of the successive slots passing station $i$ would be independent. Parallelling the argument involving Little's formula which led to (3.2) we observe that an arbitrary slot which is about to pass station $i$ is full with probability $p=\Sigma \alpha$. From the result (A.3) in the Appendix we therefore find that the conjectured limit $\tilde{L}_{i}(\infty)$ of $L_{i}(c)$ as $c \rightarrow \infty$ satisfies

$\tilde{L}_{i}(\infty)=\frac{\sigma_{i}^{2}+\alpha_{i} \sum \alpha_{j}}{2\left(1-\alpha_{i}-\sum \alpha_{j}\right)}, \quad i=1,2, \ldots, n$.

It is natural now to choose as an approximation for $L_{i}(c)$ an expression of the type

$$
\begin{aligned}
L_{i}(c) \approx & f_{i}(c) \tilde{L}_{i}(1) \\
& +\left(1-f_{i}(c)\right) \tilde{L}_{i}(\infty), \quad i=1,2, \ldots, n,
\end{aligned}
$$

where $\tilde{L}_{i}(1)=L_{i}(1)$ if an explicit expression for $L_{i}(1)$ is available and $\tilde{L}_{i}(1)$ is some approximation for $L_{i}(1)$, cf. Section 2 , if this is not the case. The functions $f_{i}(c)$, in which the parameters $n, \alpha_{j}$ and $\boldsymbol{\sigma}_{j}^{2}, j=1,2, \ldots, n$, may feature, should be decreasing and satisfying $f_{i}(1)=1$ and $f_{i}(c) \downarrow 0(c \rightarrow \infty)$. The precise form of $f_{i}(c)$ must be chosen on the basis of numerical results obtained by simulation.

If the model is completely symmetrical, so that we can write $\alpha_{i} \equiv \alpha$ and $\sigma_{i}^{2} \equiv \sigma^{2}$, one should obviously choose $f_{i}(c) \equiv f(c)$. By substitution of (2.3) and (4.1), the approximation (4.2) then reduces to

$$
\begin{aligned}
L_{i}(c) & \approx f(c) L_{i}(1)+(1-f(c)) \tilde{L}_{i}(\infty) \\
& =\frac{\sigma^{2}+n \alpha^{2}+f(c)\left(\sigma^{2}+\alpha^{2}-\alpha\right)}{2(1-(n+1) \alpha)} .
\end{aligned}
$$

It is interesting to compare (4.3) with the approximations that have appeared in the literature, since these pertain to symmetric systems. Choosing $f(c) \equiv 1$ in (4.3) we get the approximation of [13], where indeed the single-slot model is used to approximate the multi-slot model. It is reported in [13] that this approximation overestimates the exact value of $L_{i}$, as we would expect. Choosing $f(c) \equiv 0$ in (4.3) (and assuming single arrivals) we obtain the approximation of [12], which is indeed based on the assumption of independent slots. According to [12] this approximation underestimates the exact value of $L_{i}$, which is again what we would expect. It is extremely likely that a substantial improvement of these approximations should be obtained by a judicious choice of the function $f(c)$.

An extensive evaluation of approximations of the type (4.3), and, in the asymmetric case, of the type (4.2), will be reported in a later paper.

\section{Appendix}

We consider a queuing system consisting of one station at which packets arrive according to a batch Poisson process as described in Section 1. The number of packets arriving in the interval of time $[k-1, k)$ is denoted by $C_{k}, k=1,2, \ldots$ The stochastic variables $C_{1}, C_{2}, \ldots$ (denoted generically by $C$ ) are then mutually independent and identically distributed. We let $\mathscr{C}(z) \equiv E\left(z^{C}\right)$ and assume that the mean $\alpha$ and variance $\sigma^{2}$ of $C$ are finite. At each point in time $k, k=1,2, \ldots$, a 
slot passes the station. We define $D_{k}$ to be equal to 0 or 1 according to whether the $k$ th slot passing the station is empty or full, respectively. The stochastic variables $D_{1}, D_{2}, \ldots$ (denoted generically by $D$ ) constitute a sequence of mutually independent and identically distributed random variables which are also independent of the arrival process. We let $\operatorname{Pr}\{D=1\}=1-\operatorname{Pr}\{D=0)=p, 0 \leqslant p<1$. If there is at least one packet in the queue at the station and an empty slot passes the station, then exactly one packet is released from the queue, otherwise the queue length cannot decrease.

With $Q_{k}$ denoting the queue length just before the instant $k$, it is clear that $\left\{Q_{k}, k=1,2, \ldots\right\}$ constitutes an irreducible and aperiodic Markov chain which is positive recurrent if and only if $\alpha<1-p$. Moreover, we have

$Q_{k+1}=\max \left\{0, Q_{k}-\left(1-D_{k}\right)\right\}+C_{k+1}$.

Assuming steady-state conditions, it is an elementary exercise to show that (A.1) leads to

$Q(z) \equiv E\left(z^{Q}\right)=\frac{(1-p-\alpha)(1-z) \mathscr{C}(z)}{(1-p+p z) \mathscr{C}(z)-z}$

where $Q$ denotes the steady-state queue length just before the passage of a slot. Differentiating (A.2) and letting $z \uparrow 1$ subsequently yields

$E Q=Q^{\prime}(1)=\frac{\sigma^{2}+\alpha(1-\alpha)}{2(1-p-\alpha)}$.

As in the model of Section 3 , the mean queue length at an arbitrary point in time $L$ satisfies $L=E Q-\frac{1}{2} \alpha$, whence,

$L=\frac{\sigma^{2}+p \alpha}{2(1-p-\alpha)}$.
[2] B. van Arem, E.A. van Doorn and T.M.J. Meijer, Queueing analysis of a discrete closed-loop conveyor with service facilities, Queueing Systems 4 (1989) 95-114.

[3] O.J. Boxma and W.P. Groenendijk, Waiting times in discrete-time cyclic-service systems, IEEE Trans. Comm. 36 (1988) 164-170.

[4] O.J. Boxma and B. Meister, Waiting-time approximations for cyclic-service systems with switch-over times, Perform. Eval. Rev. 14 (1986) 254-262.

[5] D.J. Daley and T. Rolski, Some comparability results for waiting times in single- and many-server queues, J. Appl. Probab. 21 (1984) 887-900.

[6] W.P. Groenendijk, Waiting-time approximations for cyclic-service systems with mixed service strategies, in: Proc. 12th Int. Teletraffic Congress, Torino, Italy (1988) 1.4B.3.1-8.

[7] W.P. Groenendijk, A conservation-law based approximation algorithm for waiting times in polling systems, $R e-$ port OS-R8816, Centre for Mathematics and Computer Science, Amsterdam, The Netherlands, 1988.

[8] O.C. Ibe and X. Cheng, Approximate analysis of asymmetric single-service token-passing systems, IEEE Trans. Comm. 37 (1989) 572-577.

[9] A.E. Kamal and V.C. Hamacher, Approximate analysis of non-exhaustive multiserver polling systems with applications to local area networks, Comput. Networks ISDN Systems 17 (1989) 15-27.

[10] P.J. Kuehn, Multiqueue systems with non-exhaustive cyclic service, Bell System Tech. J. 58 (1973) 671-698.

[11] M.M. Srinivasan, An approximation for mean waiting times in cyclic server systems with nonexhaustive service, Perform. Eval. 9 (1988) 17-33.

[12] Q. Yang, D. Ghosal and I.N. Bhuyan, Performance analysis of multiple token ring and multiple slotted ring networks, in: Proc. Comput. Networking Symp., Washington (1986) 79-86.

[13] M. Zafirovic-Vukotic and I.G.M.M. Niemegeers, Performance modelling of a HSLAN slotted ring protocol, Perform. Eval. Rev. 16 (1988) 35-48.

[14] M. Zafirovic-Vukotic, I.G. Niemegeers and D.S. Valk, Performance analysis of slotted ring protocols in HSLAN's, IEEE J. Select. Areas Comm. 6 (1988) 10111024.

\section{References}

[1] B. van Arem, On stability of queueing models of local area networks with slotted ring protocols, in: Proc. IEEE Infocom '90, San Francisco (1990) 749-755. 